\title{
Cost-effectiveness of adult pneumococcal conjugate vaccination in the Netherlands
}

\author{
Marie-Josée J. Mangen ${ }^{1,12}$, Mark H. Rozenbaum ${ }^{2,3,12}$, Susanne M. Huijts ${ }^{1,4,12}$, \\ Cornelis H. van Werkhoven ${ }^{1}$, Douwe F. Postma ${ }^{1}$, Mark Atwood ${ }^{5,13}$, \\ Anna M.M. van Deursen 1,6,7,13, Arie van der Ende ${ }^{8,13}$, Diederick E. Grobbee ${ }^{1,9,13}$, \\ Elisabeth A.M. Sanders ${ }^{7,13}$, Reiko Sato ${ }^{10,13}$, Theo J.M. Verheij ${ }^{1,13}$, \\ Conrad E. Vissink ${ }^{1,13}$, Marc J.M. Bonten ${ }^{1,11,14}$ and G. Ardine de Wit ${ }^{1,14}$
}

\begin{abstract}
Affiliations: 'Julius Center for Health Sciences and Primary Care, University Medical Center Utrecht, Utrecht, The Netherlands. ${ }^{2}$ Pfizer, Capelle a/d IJssel, The Netherlands. ${ }^{3}$ Unit of Pharmacoepidemiology and PharmacoEconomics (PE2), Department of Pharmacy, University of Groningen, Groningen, The Netherlands. ${ }^{4}$ Department Respiratory Medicine, University Medical Center Utrecht, Utrecht, The Netherlands. ${ }^{5}$ Policy Analysis Inc., Brookline, MA, USA. 'Spaarne Gasthuis Academie, Spaarne Gasthuis, Hoofddorp, The Netherlands. ${ }^{7}$ Department of Pediatric Immunology and Infectious Diseases, Wilhelmina Children's Hospital, University Medical Center Utrecht, Utrecht, The Netherlands. ${ }^{8}$ Department of Medical Microbiology, Netherlands Reference Laboratory for Bacterial Meningitis, Academic Medical Center, University of Amsterdam, Amsterdam, The Netherlands. 'Julius Clinical, Academic Contract Research Organization, Zeist, The Netherlands. ${ }^{10}$ Pfizer Inc., Collegeville, PA, USA. ${ }^{11}$ Department of Medical Microbiology, University Medical Center Utrecht, Utrecht, The Netherlands. ${ }^{12}$ These authors contributed equally. ${ }^{13}$ These authors are listed alphabetically. ${ }^{14} \mathrm{~J}$ oint senior authors.
\end{abstract}

Correspondence: Marie-Josée J. Mangen, University Medical Center Utrecht, Julius Center for Health Sciences and Primary Care, Str 6.131 Room 7.115, Heidelberglaan 100, CX Utrecht 3584, The Netherlands.

E-mail: m.j.j.mangendumcutrecht.nl

ABSTRACT The Community-Acquired Pneumonia Immunization Trial in Adults (CAPiTA) demonstrated the efficacy of 13 -valent pneumococcal conjugate vaccine (PCV13) in preventing vaccinetype community-acquired pneumonia and vaccine-type invasive pneumococcal disease in elderly subjects. We examined the cost-effectiveness of PCV13 vaccination in the Netherlands.

Using a Markov-type model, incremental cost-effectiveness ratios (ICER) of PCV13 vaccination in different age- and risk-groups for pneumococcal disease were evaluated using a societal perspective. Estimates of quality-adjusted life-years (QALYs), costs, vaccine efficacy and epidemiological data were based on the CAPiTA study and other prospective studies. The base-case was PCV13 vaccination of adults aged 65-74 years compared to no vaccination, assuming no net indirect effects in base-case due to paediatric 10-valent pneumococcal conjugate vaccine use. Analyses for age- and risk-group specific vaccination strategies and for different levels of hypothetical herd effects from a paediatric PCV programme were also conducted.

The ICER for base-case was $€ 8650$ per QALY (95\% CI 5750-17100). Vaccination of high-risk individuals aged 65-74 years was cost-saving and extension to medium-risk individuals aged 65-74 years yielded an ICER of $€ 2900$. Further extension to include medium- and high-risk individuals aged $\geqslant 18$ years yielded an ICER of $€ 3100$.

PCV13 vaccination is highly cost-effective in the Netherlands. The transferability of our results to other countries depends upon vaccination strategies already implemented in those countries.

@ERSpublications

Vaccinating the elderly with 13 -valent pneumococcal conjugate vaccine is highly cost-effective http://ow.ly/NVeui

Editorial comment in: Eur Respir J 2015; 46: 1265-1268 [DOI: 10.1183/13993003.01038-2015]

This article has supplementary material available from erj.ersjournals.com

Received: Feb 262015 | Accepted after revision: May 162015 | First published online: July 092015

Copyright $\odot$ ERS 2015. ERJ Open articles are open access and distributed under the terms of the Creative Commons Attribution Non-Commercial Licence 4.0. 


\section{Introduction}

Streptococcus pneumoniae is a major cause of morbidity and mortality, with the highest rates of infection in infants, individuals with immunocompromised conditions and the elderly [1]. In many countries the 23-valent pneumococcal polysaccharide vaccine (PPV23) has been recommended for persons at high risk of pneumococcal infection, including the elderly [2]. Although recent systematic reviews and meta-analyses report protection against invasive pneumococcal disease (IPD) [2, 3], the duration of protection is limited $[4,5]$, and there is no convincing evidence that PPV23 prevents noninvasive pneumococcal community-acquired pneumonia (CAP) $[3,6]$.

Conjugated pneumococcal vaccines are effective in the prevention of IPD and CAP caused by pneumococcal vaccine-serotypes (VT) in children and elderly subjects aged $\geqslant 65$ years $[7,8]$, and prevent VT-IPD in adults with HIV [9]. Yet the cost-effectiveness of universal vaccination with the 13-valent pneumococcal conjugate vaccine (PCV13) in older adults is unknown.

Previous cost-effectiveness analyses of PCV13 suggested that vaccinating elderly and individuals with certain comorbidities might be cost-effective, but the findings were very sensitive for the assumed vaccine efficacy (VE) and other assumptions which relied on heterogenous data sources and VE estimates from expert opinions (e.g. [10-16]). Using results from the Community Acquired Pneumonia Immunization Trial in Adults (CAPiTA) and several other epidemiological studies that were performed in parallel in the Netherlands [17-20], cost-effectiveness of PCV13 vaccination of elderly and risk groups was estimated.

Methods

Model and population

A probabilistic Markov-type model [12] built using Microsoft Excel (2010; Redmond, WA, USA) with a 1 -year cycle length (see online supplementary fig. S1) was used describing the Dutch adult population from 2012 [21] in five age cohorts $(18-49,50-64,65-74,75-84$ and $\geqslant 85$ years). Each cohort is followed until death or the age of 100 years. During follow-up, subjects can transition from low- to medium- to high-risk groups (only in one direction). Similar to previous cost-effectiveness analyses, three risk groups for pneumococcal diseases were defined $[1,11,16]$ : 1) those at high risk, including individuals with an immunocompromising condition; 2) those at medium risk, including immunocompetent patients with chronic medical conditions; and 3) those at low risk, i.e. the remainder of the population (online supplementary table S1). Proportions of subjects in each risk group were based on the prevalence of clinical risk factors from electronic medical records in a large network of general practitioners (GPs) in the Netherlands (for details see section 2 of the online supplementary material).

\section{Base-case disease risk}

Age- and risk-group specific incidences of IPD were derived from recent IPD surveillance data (June 1, 2012 to May 31, 2014) from nine Dutch sentinel microbiology laboratories covering 25\% of the Dutch population. Estimates of the prevalence of comorbidities and of outcome (mortality) among individuals with IPD in the three risk groups was based on retrospective data collection, using data from 2006 to 2010 (online supplementary material, section 3.8). Based on these data, the age- and risk-group specific incidence and case fatality rates (CFRs) of IPD were estimated [22].

The incidence rate of patients hospitalised with non-IPD CAP ("inpatient CAP"), stratified by age- and risk-groups, was based on incidence of radiography-confirmed CAP admissions in the CAP-START (Study on the Initial Treatment with Antibiotics of Lower Respiratory Tract Infections) trial [18], with adjustment for coverage (i.e. proportion of International Classification of Diseases, 9th revision codes 480-486 in the CAP-START hospitals compared to the total Dutch population, using Dutch hospital data) (sections 3.2 and 3.4 of the online supplementary material). Age- and risk-group stratified CFRs of inpatient CAP were obtained from the CAPiTA and CAP-START trials [18] (sections 3.2 and 3.5 of the online supplementary material).

Age- and risk-specific incidence rates of CAP in primary care ("outpatient CAP") were based on the 2012 data from the Julius GP Network [19] (International Classification of Primary Care code R81) (section 3.7 of the online supplementary material). Incidence rates were multiplied by 0.57 to adjust for the proportion not confirmed using radiography [23]. We assumed no excess mortality risk associated with outpatient CAP [24]. To avoid double counting of costs and quality-adjusted life-years (QALY) losses, incidences of

Support statement: The model was built by Policy Analysis Inc. (PAI) on behalf of Pfizer and tested by University Medical Centre Utrecht (UMCU) and Pfizer. M. Atwood is an employee of PAI. Funding information for this article has been deposited with FundRef.

Conflict of interest: Disclosures can be found alongside the online version of this article at erj.ersjournals.com 
inpatient CAP and IPD were subtracted from incidence rates of outpatient CAP, assuming that all inpatients first consulted their GP.

The IPD surveillance data were also used to estimate the age-specific serotype distribution of IPD. Proportions of inpatient CAP attributable to vaccine serotypes in the placebo arm (10\%) were based on those episodes identified by the serotype-specific urinary antigen detection assay used in CAPiTA, without evidence of IPD. In the absence of information, similar proportions were assumed for outpatient CAP episodes.

\section{Vaccine efficacy}

VE of PCV13 for IPD and inpatient CAP in subjects in medium- and low-risk groups was based on CAPiTA [8]. In the absence of information, similar VE was assumed for outpatient CAP. The impact of age at vaccination on the VE was based on CAPiTA [25] (section 3.1 of the online supplementary material).

For subjects in the high-risk group, PCV13 VE against VT-IPD and CAP (both inpatient and outpatient) was assumed to be $22 \%$ and $35 \%$ lower, respectively, than corresponding values for the low-/medium-risk populations, based on the relative difference in VE observed in pneumococcal vaccination of children with and without HIV [26].

VE was considered stable during the first 5 years following vaccination [8], and thereafter assumed to wane annually at a rate of 5\% during years 6-10,10\% annually during years 11-15, and no efficacy was assumed from year 16 onwards. Based on CAPiTA, PCV13 vaccination was assumed not to have serious adverse effects [8].

\section{Vaccination strategy}

In the base-case scenario, the vaccination of adults aged 65-74 years with a single dose of PCV13 administered at the start of the modelling period was compared to no vaccination, consistent with the absence of universal PPV23 vaccination of the elderly in the Netherlands [27].

Vaccine coverage was assumed to be $63.9 \%$ and $81.5 \%$ in low- and medium-/high-risk subjects, respectively, similar to Dutch influenza vaccination coverage among those aged $\geqslant 65$ years in 2012 [28].

\section{Indirect effects}

PCV7 vaccination of infants was introduced in the Netherlands in 2006 and replaced by PCV10 in 2011. Incidence of IPD due to PCV7 serotypes has gradually declined in adults of all age groups since 2007, and incidence of IPD due to nonvaccine serotypes increased, especially with serotypes 19A, 7F, and 3 [22, 29]. There were no determinable indirect effects (herd protection and serotype replacement) of PCV10 vaccination in the Netherlands up to 2013 [30]. Even if PCV10 induced herd effects, serotype-replacement disease caused by 19A, 6A, and 3 could result in no net change in the preventable burden of disease of disease due to serotypes included in PCV13. Therefore, no net indirect effects of infant PCV10 vaccination was assumed for adults in the base-case, while net indirect effects for PCV7 serotypes in the seventh year after implementation were reflected in the incidence data used.

\section{Quality-adjusted life-years}

Age- and risk-group specific life expectancies were extrapolated from a previous study from the UK [11]. The QALYs lost due to premature death were calculated by multiplying the lost life-years with corresponding age-specific Dutch general population utilities [31].

Quality-of-life loss due to nonfatal inpatient CAP was derived from a case-control study nested within CAPiTA [17] (section 3.3 of the online supplementary material), using self-reported EuroQol five-dimensional (EQ-5D-3L) measurements and the Dutch EQ-5D tariff [32]. Utility loss attributable to outpatient CAP episodes was based on EQ-5D-measurements provided by the Dutch participants in an international study [20], using the Dutch EQ-5D tariff [32] (section 3.6 of the online supplementary material).

\section{Cost estimates}

The total cost per administered dose of PCV13 was $€ 79.19$, consisting of the vaccine list price (€68.56) [33] and administration costs of $€ 10.63$ [34]. Administration costs were assumed to be similar to annual influenza vaccination and included GP costs, administration and storage of vaccines [34].

Direct healthcare and non-healthcare costs were considered to start at symptom onset or first contact with the healthcare system up to a maximum 28 days or until recovery for outpatient CAP, and up to 1 month post-discharge for IPD and inpatient CAP survivors. For patients aged $<65$ years, indirect non-healthcare costs (productivity losses due to work absence from paid work) were assumed to be equal to the length of hospitalisation for nonfatal cases and 160 days (i.e. maximum friction period) for deceased cases, according to Dutch guidelines [35]. Productivity losses were based on average working hours per week and taking into account that not everybody in this age-group is working, according to Statistics Netherlands 
[36]. For hospitalised subjects aged $\geqslant 65$ years we considered productivity losses due to work absence from unpaid work of both patients and their caregivers (section 4 of the online supplementary material).

Costs were estimated by multiplying resources used, as extracted from different studies with their corresponding unit cost prices (online supplementary tables S3-S4). All costs are expressed in 2012 Euros (2012 US\$=€0.78) [37].

\section{Cost-effectiveness analysis}

The model estimated incidences of IPD, inpatient CAP, outpatient CAP, deaths, costs, life years and QALYs for scenarios with and without PCV13 vaccination. Table 1 provides an overview of the general parameters used in the model, and more details are included in online supplementary tables S5-S13 and figure S2.

Net costs, life years and QALYs gained were calculated by summing all costs, life years, and QALYs over the entire remaining lifetime of the cohort. For each scenario, 5000 model simulations were conducted using Monte Carlo sampling, accounting for the uncertainty of the model parameters (table 1).

The incremental cost-effectiveness ratio (ICER) was calculated by dividing the net cost differences between two vaccination strategies by either the life years gained (LYG) or QALYs gained. In the base-case, ICERs are reported from the societal perspective (i.e. including direct healthcare costs and non-healthcare costs) with costs discounted by $4 \%$ and health benefits by $1.5 \%$ according to Dutch guidelines [35]. In 2012, the Dutch gross domestic product (GDP) per capita was $€ 35300$ [39]. A strategy is considered highly cost-effective if ICER is $<1 \times$ GDP per capita and cost-effective if ICER is $<3 \times$ GDP per capita [40].

\section{Sensitivity and scenario analyses}

Univariate sensitivity analyses were conducted increasing/decreasing each input parameter separately by $25 \%$, keeping other parameters constant to identify critical parameters driving results. In addition, some multiway sensitivity analyses were performed. Furthermore, the impact of assumptions of VE in high-risk individuals, vaccination coverage and different discount rates on the ICER were analysed in scenario analyses (online supplementary table S14). Additionally, the impact of herd protection on the ICER was explored by decreasing the proportion of PCV13 serotypes for IPD and CAP with $0 \%$ (base-case) up to $90 \%$, with herd effects remaining stable from year 1 onwards.

We also estimated the cost-effectiveness of PCV13 vaccination of individuals aged 18-49, 18-64 and $\geqslant 50$ years as age-cohorts and considering medium- and high-risk groups only (online supplementary table S15).

\section{Results}

Effectiveness and cost-effectiveness

In the base-case scenario PCV13 vaccination would cost $€ 85.3$ million, and would prevent, on average 9850 episodes of outpatient CAP, 1850 episodes of inpatient CAP, 2050 episodes of IPD and 630 deaths during the lifetime of the cohort. This corresponds to a total gain of 5500 LYG and 4850 QALYs (discounted). As a result, PCV13 vaccination would be associated with savings of $\sim € 43.2$ million, resulting in net cost for society of $€ 42.1$ million (for more details on scenarios with and without vaccination see online supplementary table S16). The base-case ICER was $€ 8650$ per QALY (95\% CI 5750-17 100) gained or $€ 7650$ per LYG (95\% CI 5300-12450).

All vaccination strategies, except PCV13 vaccination of only low-risk patients aged 65-74 years, resulted in an ICER of $<1 \times$ GDP in $100 \%$ of the simulations (fig. 1), implying that PCV13 vaccination is highly cost-effective.

\section{Sensitivity analyses}

The ICER was most sensitive to vaccine price, duration of VE, assumed VE in high-risk individuals, VE against IPD and CFR (fig. 2, and online supplementary table S17). Yet, ICERs can be considered as cost-effective in all univariate sensitivity analyses and in the considered multiway sensitivity analyses.

PCV13 vaccination of adults aged 65-74 years (i.e. base-case) would remain cost-effective even if herd effects decreased the proportion of VT-CAP and VT-IPD by $60 \%$ (fig. 3). If vaccination costs reduced by $50 \%$ at the same time, the base-case scenario would remain cost-effective if all VT-disease were reduced by $80 \%$. When assuming only herd protection for VT-IPD, even higher reductions in incidence would remain cost-effective.

\section{Vaccination strategies}

Figure 4 depicts QALYs gained and costs for all vaccination strategies. PCV13 vaccination of high-risk individuals aged 65-74 years was cost-saving (point B). The next best alternative, gaining more QALYs at the lowest incremental costs, would be PCV13 vaccination of medium-risk persons aged 65-74 years 
TABLE 1 Overview of model input parameters in the base-case model

Variable

Population

Adult Dutch population in 2012

Distribution of population in risk-group

\section{Base-case disease risk}

IPD incidence

Inpatient CAP incidence

Outpatient CAP incidence ${ }^{+}$

Case-fatality rates for IPD

Case-fatality rates for inpatient CAP

Serotype distribution for IPD

Serotype distribution for inpatient CAP

Serotype distribution for outpatient CAP

\section{VE, waning immunity and vaccine coverage}

VE against IPD

VE inpatient CAP

VE outpatient CAP

Waning immunity

Vaccine coverage

HRQoL

Life years lost due to premature mortality

(IPD and inpatient CAP)

QALY loss due to premature mortality

(IPD and inpatient CAP)

QALY loss due to IPD

QALY loss due to inpatient CAP

\section{Costs}

QALY loss due outpatient CAP

Vaccine costs

$\mathrm{DHC}$ for IPD

DHC for inpatient CAP

DHC for outpatient CAP

DNHC for IPD

DNHC for inpatient CAP

DNHC for outpatient CAP

INHC for IPD

INHC for inpatient CAP

INHC for outpatient CAP
Expected value

Distribution

Assumption (if any) [Reference]
Age-dependent

Age- and risk-group dependent ${ }^{\S}$ Age- and risk-group dependent ${ }^{\S}$ Age- and risk-group dependent ${ }^{\S}$ Age- and risk-group dependent ${ }^{\S}$ Age- and risk-group dependent ${ }^{\S}$

Age-dependent ${ }^{\S}$

$10 \%$ of all CAP cases are VT-CAP

$10 \%$ of all CAP cases are VT-CAP

Age- and risk-group dependent ${ }^{\S}$ Age- and risk-group dependent ${ }^{\S}$ Age- and risk-group dependent $0 \%, 5 \%$ and $10 \%$

Age- and risk-group dependent ${ }^{\S}$

Age- and risk-group dependent

Age- and risk-group dependent

$$
\begin{aligned}
& 0.0709 \pm 0.020 \\
& 0.0709 \pm 0.020
\end{aligned}
$$

$0.0045 \pm 0.00051$

$€ 79.19$ per vaccinated person

Age-, risk-group and outcome dependent $^{\S}$

Age-, risk-group and outcome dependent $^{\S}$

$€ 78.25 \pm 2.54$ per case

$€ 11.9 \pm 0.5$ per fatal case and

$€ 27.7 \pm 2.9$ per survivor

$€ 11.9 \pm 0.5$ per fatal case and

$€ 27.7 \pm 2.9$ per survivor

$€ 20.26 \pm 1.87$ per case

Age-, risk-group and outcome dependent $^{\S}$

Age-, risk-group and outcome dependent $^{\S}$

18-64 years: $€ 453.01 \pm 63.33$ per case
[21]

Section 2 of the online supplementary material
Normal

Normal

$\beta$

$\beta$

$\beta$
Sentinel data $f$ CAP-START and DHC

Julius GP Network

IPD dataset

CAP-START lage < 65 years); Etio-CAP lage $\geqslant 65$ years)

Sentinel data $f$ CAPITA

Same as for inpatient CAP

$\begin{array}{ll}\beta & \text { CAPiTA [25] }{ }^{\# \#} \\ \beta & \text { CAPiTA [25 }]^{\text {ตा }}\end{array}$

$\beta \quad$ Same as inpatient CAP

Assumption, see the Methods section

[28]

[11]

Calculated based on simulated life years lost and utilities for Dutch general population \# Same as inpatient CAP

PERT $^{++}$

PERT

PERT

$\mathrm{CHO}-\mathrm{CAP}$ (age $\geqslant 65$ years); for age $<65$ years, same as for inpatient CAP (age $\geqslant 65$ years) GRACE

Vaccine [33] and administration [34] CHO-CAP; IPD dataset

CHO-CAP; pooled CAP-START and Etio-CAP

GRACE

Same as for inpatient CAP

$\mathrm{CHO}-\mathrm{CAP}$ (age $\geqslant 65$ years); for age $<65$ years, same as for inpatient CAP (age $\geqslant 65$ years)

GRACE study

CHO-CAP; IPD dataset

CHO-CAP; pooled CAP-START and Etio-CAP

GRACE

$\gamma$

Was assumed to be $€ 0$ per case for age $\geqslant 65$ years

Data are presented as mean \pm SE, unless otherwise stated. IPD: invasive pneumococcal disease; CAP: community-acquired pneumonia; VE: vaccine efficacy; HRQoL: health-related quality of life; QALY: quality-adjusted life-year; DHC: direct healthcare costs; DNHC: direct non-healthcare cost (also referred to as patient costs); INHC: indirect non-healthcare costs, i.e. productivity losses for both paid and unpaid work; CAP-START: CAP Study on the Initial Treatment with Antibiotics of Lower Respiratory Tract Infections; GP: general practitioner; Etio-CAP: Etiology of CAP study; VT: vaccine serotype; CAPiTA: Community-Acquired Pneumonia Immunization Trial in Adults; CHO-CAP: Collecting Health Outcomes and Economic Data on Hospitalized Community Acquired Pneumonia; GRACE: Genomics to Combat Resistance Against Antibiotics in Community-acquired LRTI in Europe. \#: study details can be found in the Methods section, and section 3 of the online supplementary material; ${ }^{\natural}$ : hospitalised and confirmed non-IPD CAP; ${ }^{+}$: pneumonia in primary care; ${ }^{\S}$ : data are presented in section 5 of the online supplementary material; ${ }^{f}$ : the most recent IPD surveillance data available (June 1, 2012 to May 31,2014 ) from nine Dutch sentinel microbiology laboratories covering $25 \%$ of the Dutch population [22]; \#\#: VE in the high-risk group is assumed to be $78 \%$ of corresponding values for the immunocompetent group, based on KLUGMAN et al. [26]; ๆ1: VE in the high-risk group is assumed to be 65\% of corresponding values for the immunocompetent group, based on KLugman et al. [26]; ${ }^{++}$: PERT distribution is a four-parameter $\beta$ distribution, which makes the assumption that the mean $=($ (minimum $+4 \times$ most likely + maximum) $/ 6)$. The four parameters are determined from three input values: minimum, most likely and maximum [38]. 


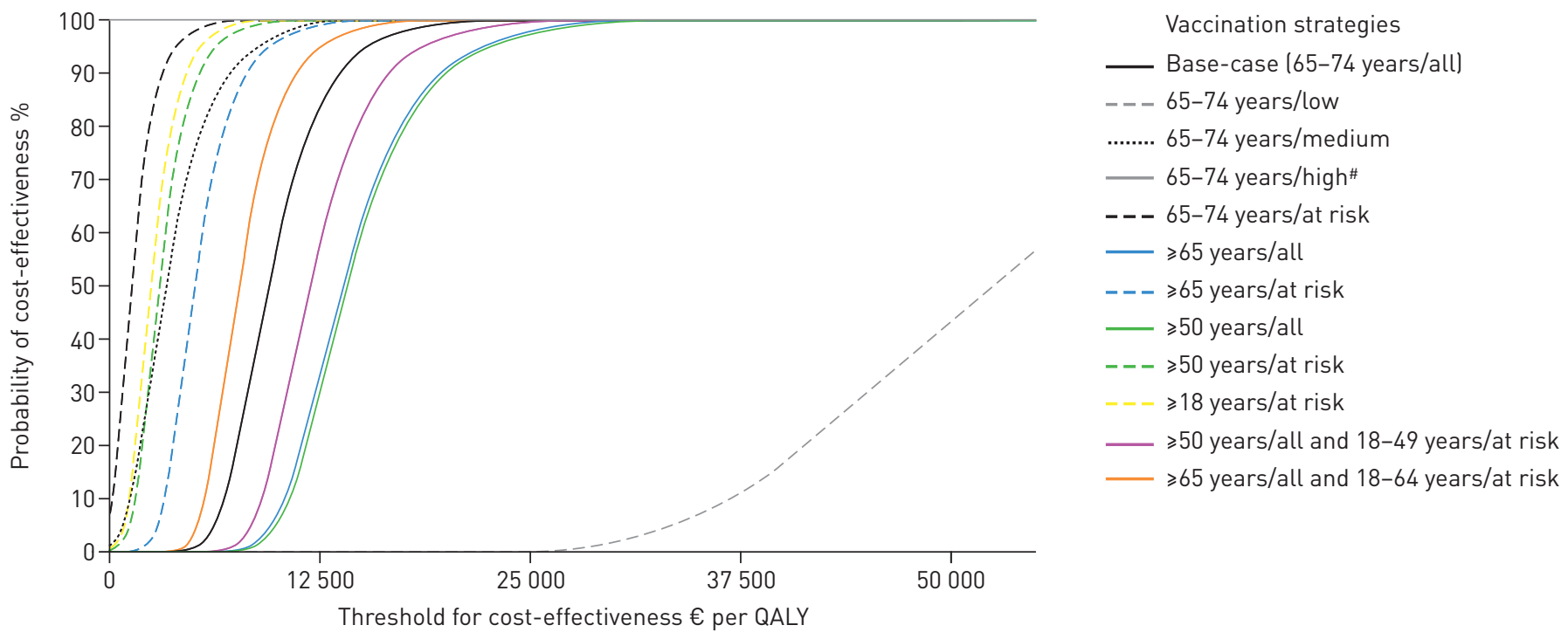

FIGURE 1 Probability of cost-effectiveness at different thresholds of willingness-to-pay for base-case and all simulated vaccination strategies using a societal perspective. Each line represents the cumulative percentage of the 5000 runs at threshold $i$. All costs are in 2012 Euros; in 2012 US $\$ 1=€ 0.78$ [37]. The gross domestic product (GDP) per capita in the Netherlands in 2012 was $€ 35300$ [36]. A strategy is considered highly cost-effective if the incremental cost-effectiveness ratio (ICER) is <1×GDP and cost-effective if ICER is <3×GDP. At risk: medium- and high risk combined. QALY: quality-adjusted life-year. ${ }^{\#}$ : $100 \%$ cost-saving (grey line at top of chart).

(point D). Vaccinating at-risk (i.e. combination of medium and high risk) adults aged $\geqslant 18$ years (point I) and vaccinating adults aged $\geqslant 50$ years combined with those at risk aged $18-49$ years (point $\mathrm{L}$ ) would be the next best alternatives. Strategies B, D and I have a $99.9 \%$ probability of being cost-effective at a societal willingness-to-pay of $€ 10000$ per QALY.

Worst-/best-case multi way SA 2

Worst-/best-case multi way SA 1

Waning immunity

VE in high-risk individuals (IPD and CAP)

Case-fatality rate (IPD and inpatient CAP)

Vaccine cost

IPD incidence

VE IPD

VE in high-risk individuals (IPD and CAP)

Discount rate used (cost and health benefit)

General mortality

VE inpatient CAP

Inpatient CAP incidence

Case-fatality rate (IPD)

DHC IPD (nonfatal)

DHC inpatient CAP (nonfatal)

Case-fatality rate (inpatient CAP)

Vaccination coverage in low-risk group

Vaccination coverage in high-risk group

General utilities for high-risk individuals

VE in outpatient CAP

Healthcare payer perspective

ICER € per QALY

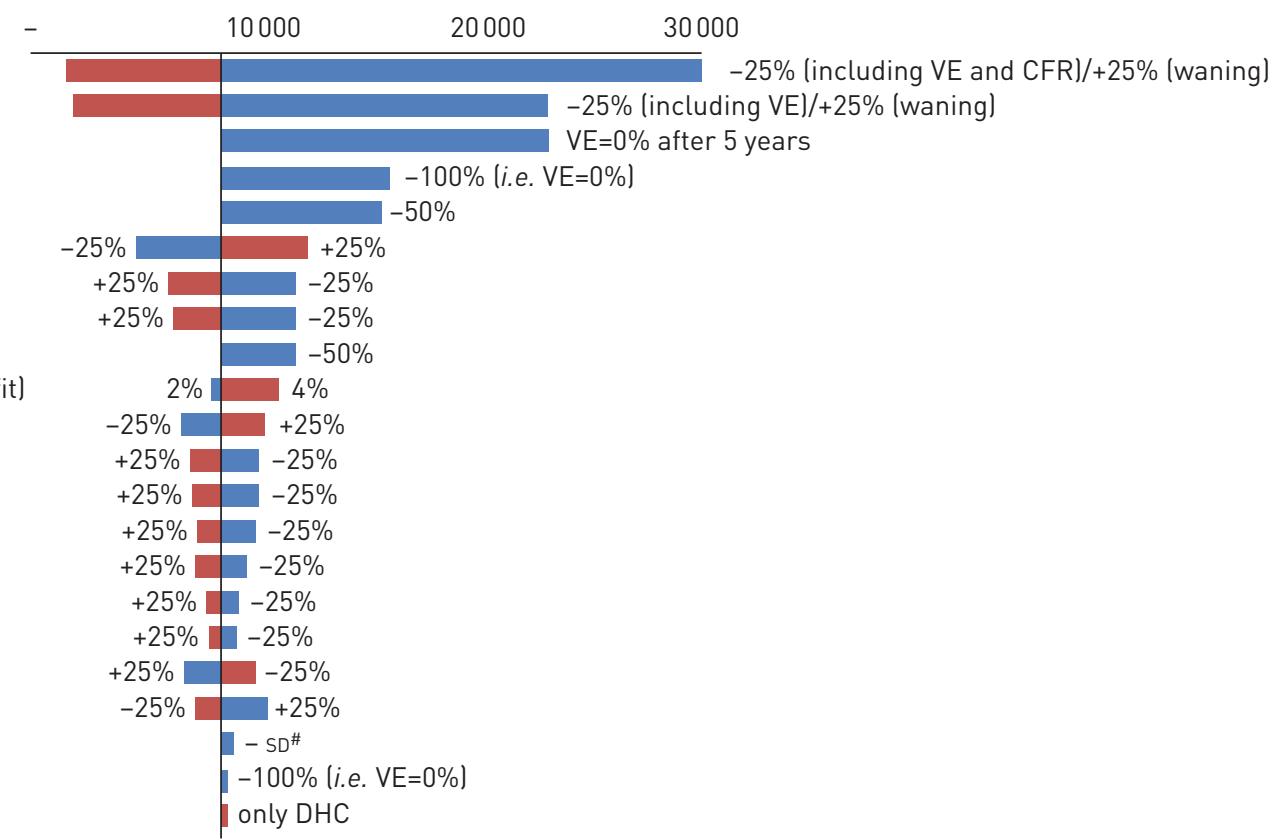

FIGURE 2 One-way sensitivity analyses, multiway sensitivity analyses and scenario analyses depicted in a tornado diagram. The $x$-axis shows the effect of changes in selected variables on the incremental cost-effectiveness ratio (ICER) ( $€$ per quality-adjusted life-year (QALY)) for the base-case taking a societal perspective. The $y$-axis shows the model parameter that was varied. The bars indicate the change in the ICER caused by changes in the value of the indicated variable holding all other parameters similar. All costs are in 2012 Euros; in 2012 US\$1=€0.78 [37]. Only results for parameters leading to an increase/decrease of $>5 \%$ of the base-case ICER are presented here. More details and results can be found in online supplementary tables S14 and S17, respectively. SA: sensitivity analysis; VE: vaccine efficacy; IPD: invasive pneumococcal disease; CAP: community-acquired pneumonia; DHC: direct healthcare costs; CFR: case fatality rate. ${ }^{\#}$ : decreasing general utilities for high-risk group by 1 sD. 


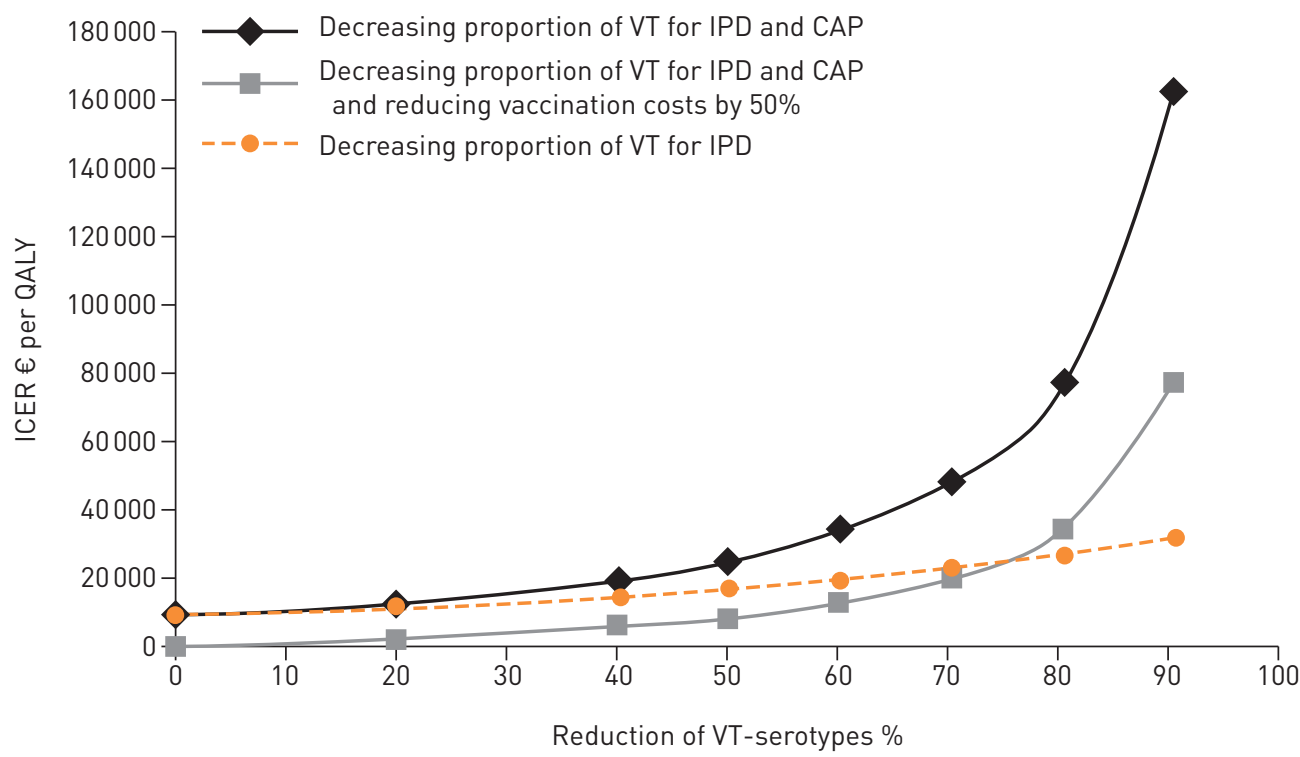

FIGURE 3 Exploring the impact of herd protection by decreasing the serotype coverage of 13-valent pneumococcal conjugate vaccine (PCV13) for invasive pneumococcal disease (IPD) and community-acquired pneumonia (CAP) from $0 \%$ (base-case) up to a maximum of $90 \%$. The orange line represents the impact of herd effects on IPD only, black and grey lines represent the impact of herd effects on IPD and CAP. The grey line represents the impact of herd protection at a $50 \%$ reduction of vaccination costs. Herd effects were assumed to be fully present at the time of initiation of PCV13 vaccination. VT: vaccine serotype; ICER: incremental cost-effectiveness ratio; QALY: quality-adjusted life-year.

\section{Discussion}

This economic evaluation, using data from recent experimental and observational studies, demonstrates that PCV13 vaccination of individuals aged 65-74 years in the Netherlands can be considered highly cost-effective, with an ICER of $€ 8650$ per QALY. This outcome was mainly driven by the favourable ICERs in high- (cost-saving) and medium-risk groups (€2874 per QALY).

Results were most sensitive to total costs of vaccination, waning immunity, CFR, VE for IPD and inpatient CAP and VE in high-risk individuals. However, the strength of the current study includes the availability of rigorously determined estimates of VE for both inpatient CAP and IPD from CAPiTA, and of QALYs lost after infection, healthcare and non-healthcare costs, and epidemiological data stratified by age- and risk-group from various recent Dutch studies [17-20]. This contrasts with earlier cost-effectiveness analyses [10-16] that used more heterogenous data sources and relied on VE estimates from expert opinion, resulting in a considerable within- and between-study variability with regard to cost-effectiveness [41].

Nevertheless, assumptions were still needed for VE in immunocompromised patients, as these individuals were not included in CAPiTA. Lower VE was assumed, based on the relative difference in VE between HIV-infected and uninfected children [26]. An assumption of no VE in immucompromised individuals would yield an ICER of $€ 16000$ per QALY. However, total absence of VE in immunocompromised individuals is unlikely, as VE of two doses of PCV7 was 74\% (95\% CI 30-90) in preventing VT-IPD in HIV-positive adults [9].

Although the VE in the CAPiTA study remained stable over an average follow-up period of almost 4 years, assumptions regarding the waning profile thereafter had to be made. Despite this uncertainty, vaccination remained highly cost-effective in a scenario analysis where the duration of protection completely disappeared after 5 years.

Age-specific VE estimates were based on a post hoc analysis of CAPiTA demonstrating a decreasing VE with increasing age [25]. However, if increasing age did not affect VE, our findings would represent underestimates of cost-effectiveness for elderly and overestimates of cost-effectiveness in younger adults.

The VE for outpatient CAP was assumed to be similar to the VE observed for inpatient CAP in the CAPiTA study. A sensitivity analysis, assuming zero VE for outpatient CAP, hardly changed the ICER (from $€ 8650$ to $€ 8900$ per QALY).

Conservatively, we included the official Dutch list price of PCV13, but vaccination costs usually reduce when implemented as a national immunisation programme and/or if administered concomitantly with annual influenza vaccination. 


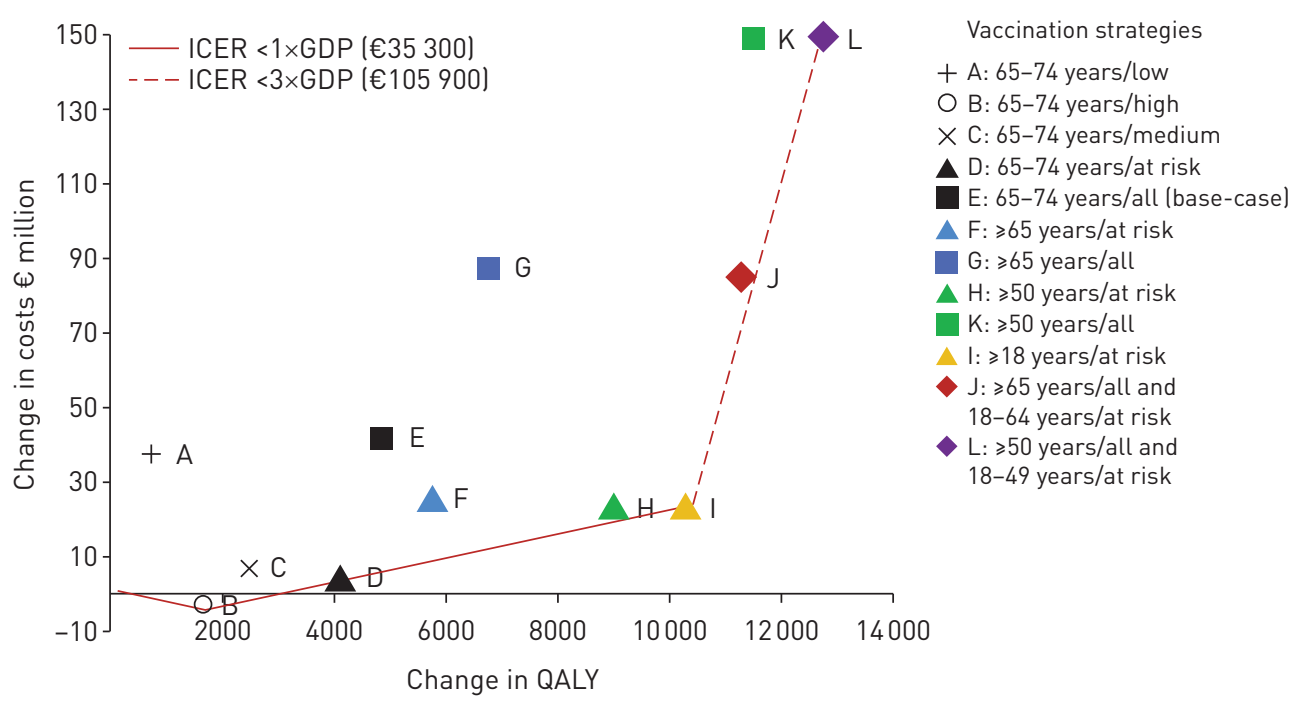

\begin{tabular}{|c|c|c|c|c|c|c|}
\hline Vaccination strategy & $\begin{array}{l}\text { Change } \\
\text { in QALY }\end{array}$ & $\begin{array}{l}\text { Change in } \\
\text { cost } € \text { million }\end{array}$ & Comparing & $\begin{array}{c}\text { Incremental } \\
\text { QALY }\end{array}$ & $\begin{array}{l}\text { Incremental } \\
\text { cost } € \text { million }\end{array}$ & ICER \\
\hline + A: 65-74 years/low & 747 & 37 & A to 0 & 747 & 37 & 50184 \\
\hline B: $65-74$ years/high & 1643 & -3 & $B$ to 0 & 1643 & -3 & Cost-saving \\
\hline X C: $65-74$ years/medium & 2474 & 7 & $\mathrm{C}$ to 0 & 2474 & 7 & 2872 \\
\hline A D: $65-74$ years/at risk & 4117 & 5 & $D$ to $B$ & 2474 & 7 & 2872 \\
\hline E: 65-74 years/all (base-case) & 4864 & 42 & $E$ to $D$ & 747 & 37 & Dominated by I \\
\hline$\Delta \mathrm{F}: \geqslant 65$ years/at risk & 5730 & 26 & $F$ to $D$ & 1613 & 22 & Dominated by I \\
\hline $\mathrm{G}: \geqslant 65$ years/all & 6771 & 87 & $G$ to $D$ & 2655 & 83 & Dominated by I \\
\hline$\Delta \mathrm{H}: \geqslant 50$ years/at risk & 9015 & 24 & $\mathrm{H}$ to $\mathrm{D}$ & 4898 & 19 & Dominated by I \\
\hline$\Delta \mathrm{I}: \geqslant 18$ years/at risk & 10286 & 24 & I to $D$ & 6169 & 19 & 3084 \\
\hline $\mathrm{J}: \geqslant 65$ years/all and $18-64$ years/at risk & 11287 & 85 & $\mathrm{~J}$ to I & 1001 & 61 & Dominated by $\mathrm{L}$ \\
\hline $\mathrm{K}: \geqslant 50$ years/all & 11496 & 149 & $\mathrm{~K}$ to I & 1210 & 125 & Dominated by $\mathrm{L}$ \\
\hline$L: \geqslant 50$ years/all and $18-49$ years/at risk & 12720 & 149 & L to I & 2434 & 125 & 51535 \\
\hline
\end{tabular}

FIGURE 4 Cost-effectiveness frontier: incremental cost-effectiveness ratios for various vaccination strategies. Points $A, B$ and $C$ are all compared to no vaccination to determine the risk group for which vaccination is the most cost-effective and the starting point for further comparison. Incremental cost-effectiveness ratio (ICER) refers to a ratio of incremental cost to incremental quality-adjusted life-year (QALY) when comparing a vaccination strategy to its next most effective alternative. Strategies lying on the curve (i.e. frontier) are more cost-effective than those lying above the curve. The latter strategies are dominated because they yield fewer QALYs at higher cost, or because the dominant strategy yields lower cost per QALY. All costs are in 2012 Euros (US\$1=€0.78). GDP: gross domestic product.

Finally, the life years lost due to premature death might have been overestimated, as hospitalised patients with CAP have a decreased long-term survival compared to the general population, and an unknown part of all 30-day mortality is attributable to comorbidities rather than to the CAP episode [42]. The use of age- and risk-group specific mortality rates partly adjusted for this. In addition, sensitivity analysis exploring $50 \%$ reduction of 30 -day CFR demonstrated that PCV13 vaccination remained highly cost-effective (i.e. $€ 15750$ per QALY).

Some model parameters were specific for the Netherlands, and may differ elsewhere. For instance, some countries, such as the USA, implemented an infant paediatric PCV13 vaccination programme, while in the Netherlands PCV10 was implemented. In the USA, PCV13 reduced IPD across all age groups including the elderly [43], while to date such effects have not been reported for the countries that implemented PCV10. During the recruitment and follow-up of CAPiTA (2008-2013), the proportion of PCV13 serotypes among elderly IPD episodes in the Netherlands decreased from $68 \%$ to $43 \%$, presumably due to indirect effects from PCV7 infant vaccination; however, the impact of paediatric vaccination on adult inpatient VT-CAP is unknown. In our base-case scenario $43 \%$ and $10 \%$ of IPD and CAP episodes, respectively, were caused by PCV13 serotypes.

The high uptake of annual influenza vaccination in the Netherlands [28] may reduce the opportunity for PCV13 vaccination benefits compared to other countries. Finally, the absence of vaccination of elderly people with PPV23 and the use of PCV10 rather than PCV13 in the paediatric programme may limit the extrapolation of our findings to other countries. Naturally, the implementation of PCV13 in the Dutch infant programme would warrant an update of the current analyses as indirect effects might reduce the 
benefits of direct vaccination. However, in our sensitivity analyses PCV13 vaccination of the elderly remained cost-effective, even with an $80 \%$ reduction in the incidence of vaccine-type infections.

\section{Acknowledgements}

We would like to thank the Genomics to Combat Resistance Against Antibiotics in Community-acquired LRTI in Europe (GRACE) team, in particular Herman Goossens of the University of Antwerp (Antwerp, Belgium; project coordinator) for allowing us to use data from the Dutch participants with regard to self-reported medical and nonmedical resources used during their illnesses, information on work absence of patients from paid work and EuroQol five-dimensional questionnaire (EQ-5D-3L) data. Alike W. van der Velden (University Medical Center Utrecht (UMCU), Utrecht, the Netherlands) is acknowledged for extracting data on primary care community-acquired pneumonia cases from the Julius GP Network. The CAPiTA (Community-Acquired Pneumonia Immunization Trial in Adults) teams in all participating hospitals are acknowledged for collecting the data and IQ-Healthcare (Nijmegen, The Netherlands) for allowing us to use their GP data. Marieke Bolkenbaas and Jan-Jelrik Oosterheert (UMCU), as well as Rogier Klok (Pfizer, Capelle a/d Ijssel, the Netherlands), are acknowledged for their critical feedback.

\section{References}

1 van Hoek AJ, Andrews N, Waight PA, et al. The effect of underlying clinical conditions on the risk of developing invasive pneumococcal disease in England. J Infect 2012; 65: 17-24.

2 World Health Organization (WHO). 23-valent pneumococcal polysaccharide vaccine. WHO position paper. Wkly Epidemiol Rec 2008; 83: 373-384.

3 Moberley S, Holden J, Tatham DP, et al. Vaccines for preventing pneumococcal infection in adults. Cochrane Database Syst Rev 2013; 1: CD000422.

4 Shapiro ED, Berg AT, Austrian R, et al. The protective efficacy of polyvalent pneumococcal polysaccharide vaccine. N Engl J Med 1991; 325: 1453-1460.

5 Andrews NJ, Waight PA, George RC, et al. Impact and effectiveness of 23-valent pneumococcal polysaccharide vaccine against invasive pneumococcal disease in the elderly in England and Wales. Vaccine 2012; 30: 6802-6808.

6 Huss A, Scott P, Stuck AE, et al. Efficacy of pneumococcal vaccination in adults: a meta-analysis. CMAJ 2009; 180 : 48-58.

7 Lucero MG, Dulalia VE, Nillos LT, et al. Pneumococcal conjugate vaccines for preventing vaccine-type invasive pneumococcal disease and X-ray defined pneumonia in children less than two years of age. Cochrane Database Syst Rev 2009; 4: CD004977.

8 Bonten MJM, Huijts SM, Bolkenbaas M, et al. Polysaccharide conjugate vaccine against pneumococcal pneumonia in adults. N Engl J Med 2015; 372: 1114-1125.

9 French N, Gordon SB, Mwalukomo T, et al. A trial of a 7-valent pneumococcal conjugate vaccine in HIV-infected adults. N Engl J Med 2010; 362: 812-822.

10 Rozenbaum MH, Hak E, van der Werf TS, et al. Results of a cohort model analysis of the cost-effectiveness of routine immunization with 13 -valent pneumococcal conjugate vaccine of those aged $\geqslant 65$ years in the Netherlands. Clin Ther 2010; 32: 1517-1532.

11 Rozenbaum $\mathrm{MH}$, van Hoek AJ, Fleming D, et al. Vaccination of risk groups in England using the 13 valent pneumococcal conjugate vaccine: economic analysis. BMJ 2012; 345: e6879.

12 Weycker D, Sato R, Strutton D, et al. Public health and economic impact of 13-valent pneumococcal conjugate vaccine in US adults aged $\geqslant 50$ years. Vaccine 2012; 30: 5437-5444.

13 Kuhlmann A, Theidel U, Pletz MW, et al. Potential cost-effectiveness and benefit-cost ratios of adult pneumococcal vaccination in Germany. Health Econ Rev 2012; 2: 4.

14 Jiang Y, Gauthier A, Keeping S, et al. Cost-effectiveness of vaccinating the elderly and at-risk adults with the 23-valent pneumococcal polysaccharide vaccine or 13-valent pneumococcal conjugate vaccine in the UK. Expert Rev Pharmacoecon Outcomes Res 2014; 14: 913-927.

15 Smith KJ. Determining the cost-effectiveness of adult pneumococcal vaccination strategies. Expert Rev Pharmacoecon Outcomes Res 2014; 14: 1-4.

16 Smith KJ, Wateska AR, Nowalk MP, et al. Cost-effectiveness of adult vaccination strategies using pneumococcal conjugate vaccine compared with pneumococcal polysaccharide vaccine. JAMA 2012; 307: 804-812.

17 Mangen MJ, Bonten MJ, de Wit GA. Rationale and design of the costs, health status and outcomes in community-acquired pneumonia (CHO-CAP) study in elderly persons hospitalized with CAP. BMC Infect Dis 2013; 13: 597.

18 Postma DF, van Werkhoven $\mathrm{CH}$, van Elden LJR, et al. Antibiotic treatment strategies for community-acquired pneumonia in adults. $N$ Engl J Med 2015; 372: 1312-1323.

19 van den Broek d'Obrenan J, Verheij TJ, Numans ME, et al. Antibiotic use in Dutch primary care: relation between diagnosis, consultation and treatment. J Antimicrob Chemother 2014; 69: 1701-1707.

20 van Vugt SF, Broekhuizen $\mathrm{BD}$, Lammens $\mathrm{C}$, et al. Use of serum $\mathrm{C}$ reactive protein and procalcitonin concentrations in addition to symptoms and signs to predict pneumonia in patients presenting to primary care with acute cough: diagnostic study. BMJ 2013; 346: f2450.

21 Centraal Bureau voor de Statistiek. Bevolking; geslacht, leeftijd, burgerlijke staat en regio, 1 januari [Population; sex, age, marital status and region, January 1]. Heerlen, Statistics Netherlands (CBS), 2014. http://statline.cbs.nl/ Statweb/publication/?DM=SLNL\&PA=03759ned \&D1=0\&D2=0,19-96,118,129\&D3=0\&D4=24\&HDR=T\&STB=G2, G3,G1\&VW=T Date last accessed: December 4, 2014.

22 van Deursen AM, van Mens SP, Sanders EA, et al. Invasive pneumococcal disease and 7-valent pneumococcal conjugate vaccine, the Netherlands. Emerg Infect Dis 2012; 18: 1729-1737.

23 van Vugt SF, Verheij TJ, de Jong PA, et al. Diagnosing pneumonia in patients with acute cough: clinical judgment compared to chest radiography. Eur Respir J 2013; 42: 1076-1082.

24 Bont J, Hak E, Hoes AW, et al. Predicting death in elderly patients with community-acquired pneumonia: a prospective validation study reevaluating the CRB-65 severity assessment tool. Arch Intern Med 2008; 168: $1465-1468$. 
van Werkhoven $\mathrm{CH}$, Huijts SM, Bolkenbaas $\mathrm{M}$, et al. 13-valent pneumococcal conjugate vaccine efficacy is declining with old age: results from an exploratory analysis of the CAPiTA trial. ID-Week, Philadelphia, October 8-12, 2014. Abstract 1099. https://idsa.confex.com/idsa/2014/webprogram/Paper47255.html

26 Klugman KP, Madhi SA, Huebner RE, et al. A trial of a 9-valent pneumococcal conjugate vaccine in children with and those without HIV infection. N Engl J Med 2003; 349: 1341-1348.

27 Health Council of the Netherlands. Pneumococcal vaccine in elderly adults and risk groups. The Hague, Health Council of the Netherlands; publication no. 2003/10; 2003. www.gezondheidsraad.nl/sites/default/files/0310n.pdf Date last accessed: June 25, 2015.

28 Jansen B, Tacken M, Mulder J, et al. Monitoring vaccination rate - Dutch National Influenza Prevention Program - 2012. Nijmegen, UMC St Radboud, Scientific Institute for Quality of Healthcare, 2013. www.iqhealthcare.nl/ media/73940/kort_npg_monitoring_2012_def_met_isbn.pdf Date last accessed: June 25, 2015.

29 Lexau CA, Lynfield R, Danila R, et al. Changing epidemiology of invasive pneumococcal disease among older adults in the era of pediatric pneumococcal conjugate vaccine. JAMA 2005; 294: 2043-2051.

30 Knol MJ, Sanders EA, Vlaminckx B, et al. Incidence of invasive pneumococcal disease in the Netherlands after introduction of 7-valent and 10-valent pneumococcal conjugate vaccination. Pneumonia 2014; 3: 0261.

31 Stolk E, Krabbe P, Busschbach J. Using the Internet to collect EQ-5D norm scores a valid alternative? In: Busschbach J, Rabin R, De Charro F, eds. 24th Scientific Plenary Meeting of the EuroQol Group - Proceedings:153-1K5. Rotterdam, EuroQol Group, 2009.

32 Lamers LM, McDonnell J, Stalmeier PF, et al. The Dutch tariff: results and arguments for an effective design for national EQ-5D valuation studies. Health Econ 2006; 15: 1121-1132.

33 Z-Index. Official Dutch Drug Prices. Den Haag, Z-Index BV, 2014. www.z-index.nl/ Date last accessed: September 17, 2014.

34 SNP. Grieppreventie SNP - Declareren/ opgave vaccinaties doen. Utrecht, Stichting Nationaal Programma Grieppreventie (SNP), 2014. www.snpg.nl/griepcampagne/declareren.htm Date last accessed: April 28, 2014.

35 Tan SS, Bouwmans CA, Rutten FF, et al. Update of the Dutch Manual for Costing in Economic Evaluations. Int J Technol Assess Health Care 2012; 28: 152-158.

36 CBS. Working population; numbers according to gender and other person characteristics. 2014 http://statline.cbs.nl/ StatWeb/publication/?DM=SLNL\&PA=71958ned\&D1=0-1,4\&D2=0\&D3=0,2-11\&D4=65\&HDR=T\&STB=G1,G2,G3\& VW=T Date last accessed: August 8, 2014.

37 OECD. Exchange Rates (indicator), 2015. doi: 10.1787/037ed317-en Date last accessed: June 26, 2015.

38 Vose D. Risk Analysis: a Quantitative Guide. Chichester, John Wiley \& Sons Ltd, 2000.

39 OECD. National Accounts at a Glance 2014. Paris, The Organisation for Economic Co-operation and Development (OECD). http://stats.oecd.org/index.aspx?queryid=28226 Date last accessed: June 25, 2015.

40 Tan-Torres Edejer T, Baltussen R, Adam T, et al. Making choices in health: WHO guide to cost-effectiveness analysis. Geneva, World Health Organization, 2003. www.who.int/choice/publications/p_2003_generalised_cea.pdf

41 van de Vooren K, Duranti S, Curto A, et al. Cost effectiveness of the new pneumococcal vaccines: a systematic review of European studies. Pharmacoeconomics 2014; 32: 29-45.

42 Restrepo MI, Faverio P, Anzueto A. Long-term prognosis in community-acquired pneumonia. Curr Opin Infect Dis 2013; 26: 151-158.

43 Moore MR, Link-Gelles R, Schaffner W, et al. Effect of use of 13-valent pneumococcal conjugate vaccine in children on invasive pneumococcal disease in children and adults in the USA: analysis of multisite, population-based surveillance. Lancet Infect Dis 2015; 15: 301-309. 